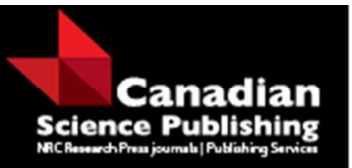

Canadian Journal of Physiology and Pharmacology Revue canadienne de physiologie et pharmacologie

\title{
Implementation of the mouse frailty index
}

\begin{tabular}{|r|l|}
\hline Journal: & Canadian Journal of Physiology and Pharmacology \\
\hline Manuscript ID & cjpp-2017-0025.R1 \\
\hline Manuscript Type: & Review \\
\hline Date Submitted by the Author: & 08-Feb-2017 \\
\hline & $\begin{array}{l}\text { Kane, Alice; Dalhousie University, } \\
\text { Ayaz, Omar; Dalhousie University } \\
\text { Ghimire, Anjali; Dalhousie University } \\
\text { Feridooni, Hirad; Dalhousie University } \\
\text { Howlett, Susan; Dalhousie University, Dept. of Pharmacology }\end{array}$ \\
\hline Keyword: & frailty index, deficit accumulation, deficit index, aging, preclinical models \\
\hline \multicolumn{2}{|c}{} \\
\hline
\end{tabular}

SCHOLARONE ${ }^{m}$

Manuscripts 


\title{
Implementation of the mouse frailty index
}

\author{
Alice E. Kane ${ }^{1}$, Omar Ayaz ${ }^{1}$, Anjali Ghimire ${ }^{1}$, Hirad A. Feridooni ${ }^{1}$, Susan E. Howlett ${ }^{1}$
}

\author{
Affiliations: \\ ${ }^{1}$ Pharmacology Department, Dalhousie University, Halifax, NS, Canada
}

\section{Running Head:}

Implementation of the mouse FI

\section{Corresponding Author:}

Susan Howlett, PhD

Department of Pharmacology

Level 6, Sir Charles Tupper Medical Building

PO Box 15000

Halifax, Nova Scotia

Canada B3H 4R2

Phone: +1 9024943552

Email: susan.howlett@dal.ca 


\section{ABSTRACT}

Frailty is considered a state of high vulnerability for adverse health outcomes for people of the same age. Those who are frail have higher mortality, worse health outcomes and use more health care services than those who are non-frail. Despite this, little is known about the biology of frailty, the effect of frailty on pharmacological or surgical outcomes and potential interventions to attenuate frailty. In humans, frailty can be quantified using a frailty index (FI) based on the principle of deficit accumulation. The recent development of an FI in naturally ageing mice provides an opportunity to conduct frailty research in a validated preclinical model. The mouse FI has been successfully used across a wide range of applications, however there are some factors that should be considered in implementing this tool. This review summarises the current literature, presents some original data and suggests areas for future research on the current applications of the mouse FI, inter-rater reliability of the FI, the effect of observer characteristics and environmental factors on mouse FI scores, and the individual items that make up the FI assessment. The implementation of this tool into preclinical frailty research should greatly accelerate translational research in this important field.

Key Words: frailty index, deficit index, deficit accumulation, aging, preclinical models, mouse models, translational 


\section{Introduction}

The term "frailty" is widely used in the clinical literature to describe a state of increased vulnerability to adverse health outcomes in older adults (Rockwood et al. 1994). Frailty is an important clinical challenge, as frail individuals have higher mortality, worse outcomes and use more health care services than non-frail individuals of the same age (Rockwood et al. 2011). Still, little is known about the biological basis of frailty, in part because it is difficult to work with this vulnerable and heterogeneous clinical population.

An important advance in the field has been the development of novel tools to quantify frailty in naturally aging animals, as highlighted in several recent reviews (Howlett 2015; Kane et al. 2016; Kane and Howlett 2017; Seldeen et al. 2015; Zglinicki et al. 2016). This review will focus on the mouse clinical frailty index (FI), a recently developed, non-invasive frailty assessment tool that was adapted from an instrument widely used in clinical studies. Although the mouse clinical FI has been successfully used by a number of research groups, there are important factors that should be considered in implementing this tool. This review addresses some of these factors, including the inter-rater reliability of the index, the effect of the experience, sex and background of the person completing the FI, the effect of the location and timing of the FI assessment, the effect of animal housing conditions, and the individual items included in the FI assessment. We summarise available literature, suggest areas for future experimentation and present some original data.

\section{Frailty and its assessment}

Frailty is considered a state of high vulnerability for adverse health outcomes and it is associated with aging (Clegg et al. 2013; Collard et al. 2012). Although there are many different 
instruments that have been used to evaluate frailty in clinical studies (Buta et al. 2016), one commonly used approach is to consider frailty as a state that can be quantified with a FI based on deficit accumulation (Mitnitski et al. 2001). The FI tool assesses the number of health-related deficits a person has accumulated, with a focus on the number of deficits, rather than the specific nature of the deficits (Rockwood and Mitnitski 2007). Health-related deficits can include comorbidities, abnormal laboratory results, self-reported functional deficits and symptoms (Howlett et al. 2014; Mitnitski et al. 2002; Rockwood and Mitnitski 2007). The number of health-related deficits a person displays is counted, and divided by the maximum number of deficits measured, to give an FI score, theoretically between 0 and 1. Clinical studies have shown that higher FI scores are associated with adverse outcomes such as increased risk of hospitalisation, institutionalisation, falls, and death in a variety of older adult populations (Collard et al. 2012; Mitnitski et al. 2002). However, little is known about the mechanisms underlying the development of frailty, the effect of frailty on specific outcomes (e.g. drug metabolism, surgical recovery), and potential interventions to attenuate frailty (Hubbard and Theou 2012). One reason for this is that research into frailty is limited by the logistical and ethical difficulties of either interventional or observational studies in the heterogeneous and dependent older, frail population (Harris and Dyson 2001). The recent development of a novel approach to quantify frailty with a FI in naturally ageing mice provides an opportunity to conduct frailty research in a validated preclinical model.

The mouse FI approach was first developed by Parks et al. (2012). They assessed 31 health-related items in middle-aged (12 months) and old (30 months) male and female C57BL/6 mice. The items included hemodynamic measurements (eg. diastolic blood pressure, heart rate), activity measures (eg. total distance moved, rearing frequency in an open field), measurements of 
basic metabolism (eg. blood sodium, glucose), and body composition (eg. bone mass density, percent body fat). Each potential health deficit was scored based on how many standard deviations (SD) the measured value was from the mean reference values obtained in middle-aged animals. For example, if a result was $1 \mathrm{SD}$ from the mean it was scored 0.25 , and if it was more than 4 SDs from the mean it was given a score of 1 . The sum of these scores was divided by the maximum number of deficits measured to give a FI score between 0 and 1 for each mouse. They found that mean FI scores increased with age in a similar way in male and female mice, and that increasing FI score was associated with detrimental age-related changes in the heart, including cardiomyocyte hypertrophy and contractile dysfunction (Parks et al. 2012).

Whitehead et al. (2014) continued this work with the development of a mouse FI based on simply assessed, non-invasive 'clinical' deficits in health. This clinical FI assessment can be completed in several minutes, and requires no specialised equipment. It assesses 31 different deficits across a range of domains including the integument, musculoskeletal system, vestibulocochlear/auditory systems, ocular and nasal systems, digestive system, urogenital system, respiratory system, signs of discomfort, as well as the body weight, and body surface temperature. The individual items included in the index are shown in Table 1. Most items were given a score of 0 in the absence of a health deficit, 0.5 if the health deficit is mildly present, and 1 if the deficit is severely present. The weight and temperature were scored in 0.25 units as described in Parks et al. (2012), based on how many SDs the item measured was from mean reference values in young animals (Table 1). FI scores were determined for male and female C57BL/6 mice aged 5, 19 and 28 months. As reported by Parks et al. (2012), they found an increase in mouse clinical FI scores with age in mice of both sexes. Whitehead et al. (2014) also compared FI scores obtained in mice with scores in humans across a range of ages. When frailty 
scores were normalised to the age of $90 \%$ mortality in mice and in humans, they found that deficits accumulated at a very similar rate in the two groups. In addition, for any specific normalised age, mice and humans had very similar FI scores.

More recently, the characteristics of the FI have been compared directly between humans and mice. Rockwood et al. (2017) assessed frailty in 251 male C57BL/6 mice at several times over their lifespan (eg. 1, 6, 12, 18 months). They compared this data to human FI data from the large National Health and Nutrition Examination Survey (NHANES) dataset. They found that, as in humans, increasing FI scores in mice significantly predicted the risk of mortality. Additionally, the rate of deficit accumulation, the submaximal limit of the FI, and the distribution of FI scores with age were very similar in mice and humans (Rockwood et al. 2017). These data demonstrate that a FI based on clinically apparent health deficits can be used to characterize frailty in aging mice, and the characteristics are similar to a human FI.

\section{Applications of the mouse clinical FI}

The mouse clinical FI provides a validated preclinical model to research frailty across a wide range of applications. The potential research applications of this tool were recently reviewed (Kane et al. 2016b). Possible uses include exploring the effect of interventions to delay or prevent frailty and exploring the effect of frailty on a range of outcomes including drug toxicity or pharmacokinetics and surgical outcomes. Furthermore, the FI tool can be used to investigate the biology and mechanisms of frailty, and it can help identify frailty biomarkers. Indeed, since the first publications of the mouse FI (Parks et al. 2012; Whitehead et al. 2014) several studies have already used this tool to investigate the impact of interventions on frailty and the effect of frailty on outcomes. These have been recently reviewed (Kane et al. 2016b; Kane and Howlett 2017; Seldeen et al. 2015; Zglinicki et al. 2016), and are discussed below. 
Kane et al. (2016a) was the first study to explore potential frailty interventions using the mouse clinical FI. They investigated the effect of known longevity interventions, specifically the polyphenol resveratrol and the dietary intervention of calorie restriction, on frailty in mice. These interventions have previously been shown to increase lifespan in mouse strains (Baur et al. 2006; Weindruch et al. 1986), and the authors aimed to investigate whether they also attenuated frailty in mice. Resveratrol was administered in the diet at a dose of $100 \mathrm{mg} / \mathrm{kg} /$ day to male $\mathrm{C} 57 \mathrm{BL} / 6$ mice aged 19 months of age for 6 months. They found that this chronic exposure to resveratrol, even when started late in life, attenuated frailty. They only investigated the effect in male mice, so whether this effect is also seen in females would be an interesting future study. For the calorie restriction study, both male and female $\mathrm{C} 57 \mathrm{BL} / 6$ and $\mathrm{DBA} / 2 \mathrm{~J}$ mice were exposed to calorie restriction (40\% less than ad libitum fed) from 6 months of age, until 19 months. The authors found that lifelong calorie restriction attenuated frailty in male C57BL/6 mice, and saw a trend to this effect in females. They also found that the DBA/2J mice, which is a short lived mouse strain (Yuan et al. 2011), had higher FI scores than the C57BL/6 mice at the same chronological age (Kane et al. 2016a). These studies clearly demonstrate that the mouse FI tool can be used to evaluate interventions that either attenuate or promote frailty.

Huizer-Pajkos et al. (2016) used the mouse clinical FI to assess the effect of polypharmacy on frailty. Polypharmacy, which is exposure to 5 or more different medications, is very common in the older population, and in observational clinical studies has been linked to an increased risk of frailty (Gnjidic et al. 2012). Studies in humans, however, are confounded by the heterogeneity of the older population including multiple diseases and diverse multiple medications. In order to better understand the risks of polypharmacy, the authors developed a mouse model that would allow investigation of the effect of polypharmacy on outcomes in a 
controlled preclinical study. This model of polypharmacy involved exposing young (14 weeks) and old (99 weeks) male C57BL/6 mice to 5 commonly used medications in their diet (acetaminophen, simvastatin, metoprolol, omeprazole and citalopram). They assessed the effect of 2 weeks polypharmacy exposure on a range of clinically-relevant geriatric outcomes including FI, grip strength and gait speed. The authors found that, in old mice but not in young, polypharmacy resulted in declines in functional outcomes such as decreased locomotor activity and a trend towards increased frailty. The authors suggest that the lack of significance in this result may be due to the short intervention time frame; changes in items in the mouse clinical FI, such as muscoskeletal changes, may take longer than 2 weeks to become apparent (HuizerPajkos et al. 2016). Further studies in this mouse model could increase understanding of the relationship between polypharmacy and frailty, as well as shed light on the potential mechanisms involved.

Kane et al. (2016c) assessed the effect of frailty on acetaminophen toxicity risk in old mice. Old age and frailty are associated with physiological changes that can affect the toxicology of drugs such as acetaminophen, as well as a high prevalence of adverse drug reactions (Burgess et al. 2005; Mitchell et al. 2011). Despite this there are very few clinical studies on changing risks of drug toxicity in older people. As such, the authors of this study aimed to use the mouse clinical FI to investigate the risk of acetaminophen toxicity in old age and frailty. They treated young and old male C57BL/6 mice with three different exposures to acetaminophen (chronic, sub-acute and acute) either given in the diet, or orally-gavaged. They found no association between increased risk of toxicity and old age or frailty, over any of the exposure types. The authors did identify, however, a significant association between higher FI scores, and low levels 
of total protein, albumin and alkaline phosphatase in serum, and suggest these could be potential frailty biomarkers (Kane et al. 2016c).

Langille et al. (2016) used the mouse clinical FI to explore the effect of old age and frailty on changes in the gut microbiome. As with clinical polypharmacy studies, clinical studies of the microbiome have been confounded by the heterogeneity of the older population, including varied diets and places of residence. Therefore, the authors of this study aimed to explore the association of age and frailty with the gut microbiome using the mouse clinical frailty index. The authors collected stool samples from female C57BL/6 mice aged 174-857 days and completed metagenomics analysis to compare differences in the taxonomic composition and functional differences of the microbiome with age and frailty. They found that both old age and frailty were associated with clinically-relevant changes such as reduced function of lactate-utilising bacteria, which is associated with inflammatory bowel diseases, and increased beta-glucuronidases which have a role in drug-induced toxicity. These changes could have implications for nutrition and pharmacology in old age and frailty (Langille et al. 2016).

Moghtadaei et al. (2016) explored the association of frailty with changes in heart rate and sinoatrial node function in mice. They assessed frailty in young (14 weeks) and old (102 weeks) male C57BL/6 mice, using the mouse clinical FI. They then completed in vivo electrophysiology experiments on the mouse hearts and high resolution optical mapping of the atria, to investigate changes in sinoatrial node function as well as the underlying mechanisms. They found that sinoatrial node function was impaired in aging mice. Interestingly, they also found that detrimental age-related changes including increased atrial fibrosis, changes in electrical conduction and decreased heart rate, were correlated with and graded by frailty. The authors concluded that mice of the same chronological age had variability in their health status as 
quantified with the FI tool and that frailty was an important predictor of atrial dysfunction in aging mice (Moghtadaei et al. 2016).

Together these studies demonstrate that the mouse clinical FI is a valuable tool for preclinical frailty research across a range of applications. This preclinical model of frailty is an important development that will help investigate mechanisms involved in frailty, facilitate the development of interventions to modify frailty, and allow exploration of the impact of frailty on interventions.

\section{Inter-rater reliability of mouse clinical FI}

For any assessment tool, it is important to demonstrate that the instrument is reliable when used by different assessors. The degree of agreement among raters is typically assessed with statistics, such as the intra-class correlation coefficient (ICC). The inter-rater reliability of the mouse clinical FI was first investigated by Feridooni et al. (2015). They had two different scientists (raters) assess 233 middle-aged male C57BL/6 mice over three different time points. After each time point, scoring was compared and refinements were discussed. The inter-rater reliability for the first time point was very good $(\mathrm{ICC}=0.51)$, although it improved over the three time points, and by the final time point the ICC was 0.77 , which represents excellent inter-rater reliability (Streiner et al. 2015). Feridooni et al. (2015) suggested refinements to the descriptors provided in the original mouse clinical FI paper (Whitehead et al. 2014) for individual deficits including temperature and hearing loss. In particular, they emphasized that the clicking sound used for the hearing loss assessment needed to be novel, so they suggested exposing only one mouse at a time to the noise. In addition, it is important to be consistent with respect to the abdominal placement of the temperature probe (Feridooni et al. 2015). These refinements to the deficit descriptors may improve the inter-rater reliability for future users of the mouse clinical FI. 
Kane et al. (2015) also investigated the inter-rater reliability of the mouse clinical FI. They presented ICCs from several experiments with two scientist raters and found that there was good to excellent agreement between raters in all experiments. These results are particularly important as these assessments were completed by a different research group (in a different country), but the results were very similar to those reported by Feridooni et al. (2015). The authors did note that, without discussion, ICCs did not improve between raters over time. This clearly demonstrates the importance of discussing and refining assessment techniques if using more than one rater for the mouse clinical FI.

We also present here some new data on inter-rater reliability of the mouse clinical FI. Four scientist raters: one female inexperienced with the mouse clinical FI assessment, one female with experience, one inexperienced male and, one experienced male, assessed the FI in 45 mice over a 4 week period, with all raters assessing all mice. There was an initial discussion and training session between all four raters, and then assessments were completed by each rater individually and independently. Despite the differences between the raters, we found excellent inter-rater reliability between all four raters $(\mathrm{ICC}=0.80$, Table 2$)$. We also found excellent interrater reliability when those of different experience levels $(\mathrm{ICC}=0.76)$ or different sexes $(\mathrm{ICC}=0.86)$ were compared directly (Table 2).

Overall the results from these three studies show that the mouse clinical FI exhibits a high degree inter-rater reliability when used by a variety of types of raters in different research groups. How the specific characteristics of the assessor, and/or the assessment conditions may affect the mouse clinical FI, are discussed in the next sections.

\section{Effect of observer on mouse clinical FI}


It is possible that specific characteristics of the raters themselves can affect the assessment of experimental animals. For example, Sorge et al. (2014) demonstrated that the sex of the person assessing mice for pain affects the measured outcomes. They showed that mice who were assessed for pain by men, or even who were exposed to clothes worn by men, had stress-induced pain inhibition compared to those assessed by women (Sorge et al. 2014). These observations suggest that the sex of the observer may be important to consider in the assessment of mouse frailty with the clinical FI tool. To investigate this possibility, C57BL/6 mice across three age groups (young=38.7 \pm 2.3 weeks, $n=15$; middle-aged=60.7 \pm 5.4 weeks, $n=24$; old=101.4 \pm 19.4 weeks, $n=6$ ) were assessed for the mouse clinical FI by 2 female and 2 male assessors. Assessments were completed in separate rooms, and there were at least 3 days between assessments on the same mice by people of different sexes. Mean FI scores were calculated for the different mouse age groups for observers of different sexes (Figure 1A). A 2way ANOVA showed a significant increase in mean FI with increasing age $(p<0.001)$, but no effect of observer sex $(p=0.84)$ on mean FI score. Figure 1B shows that there was extremely good correlation between FI scores from male observers and female observers $(r=0.70$, $p<0.0001$, and there was also excellent correlation as assessed by the inter-rater correlation coefficient (Table 2). Overall, these results suggest that the sex of the rater is not a significant factor in completing the mouse clinical FI assessment.

Another potential factor that could affect FI scoring is the degree of experience a person has with the assessment tool. In the same experiment described above, the effect of observer experience on mouse clinical FI scoring was investigated. Two raters (one male, one female) with several years' experience using the mouse clinical FI tool and two raters (one male, one female) who had no prior experience using the mouse clinical FI tool, participated in completing 
the FI scoring for young, middle-aged, and old C57BL/6 mice, as described above. Figure 2A shows that there was no effect of observer experience $(p=0.47)$ on mean FI score. Interestingly, there was good correlation between FI scores from experienced and inexperienced observers $(r=0.75, p<0.0001)$ (Figure $2 \mathrm{~B})$. These results indicate that the experience of the rater with the mouse clinical FI does not affect FI scores. On the other hand, a discussion session between raters, if more than one rater is to be used for frailty assessments, appears to be beneficial.

Although the specific experience of the observer with the mouse clinical FI does not influence FI scores, Kane et al. (2015) have shown that the professional background or training of the observer does appear to affect scoring. They assessed the inter-rater reliability of four raters assessing $74 \mathrm{C} 57 \mathrm{BL} / 6$ mice $(19.0 \pm 1.0$ months $)$ for frailty with the mouse clinical FI. Two of the raters were scientists with experience in animal research, and two raters were animal technicians with extensive training in animal husbandry. They found that agreement was high between raters of the same professional background $(\mathrm{ICC}=0.88)$. By contrast, inter-rater reliability was poor between those of different backgrounds (ICC $=0.20-0.49)$. The highest level of agreement was seen between the two scientist researchers. Based on these observations, Kane et al. (2015) recommend that care be taken in selecting raters for the index, especially if the results are to be compared across raters. It is important to note that the present study, and prior studies exploring inter-rater reliability of the mouse clinical FI (Feridooni et al. 2015), used research scientists as raters.

Other characteristics of the rater/observer could, in theory, affect operationalization of the mouse clinical FI, although these have not yet been investigated. It is conceivable that bias of researchers towards their own hypotheses may have an effect. Therefore, as with all scientific assessments, mouse clinical FI assessments should be performed by blinded raters whenever 
feasible (Macleod et al. 2015). It has also been shown that the specific handling of mice by a rater can affect outcomes of studies of cognitive function, with mice handled by the tail appearing more anxious than those exposed to cupped handling (Novak et al. 2015); it would be interesting to see if this also can impact the assessment of frailty in the mouse.

Overall, the original results presented in this section demonstrate that neither the sex nor the rater's prior experience using the mouse FI assessment tool effects mouse clinical FI scoring. One study suggests that the professional background of the rater does appear to effect mouse clinical FI scoring, and it would be interesting to look at the effect of other rater characteristics on the operationalisation of the mouse clinical FI.

\section{Environmental factors that may affect mouse FI scores}

Along with considerations regarding the assessor as outlined above, environmental factors should be considered when implementing the mouse clinical FI. Each of the initial publications on the mouse clinical FI (Feridooni et al. 2015; Parks et al. 2012; Whitehead et al. 2014) specify that the assessments be completed in a quiet, separate room. They also emphasize that the mice be allowed to acclimatise to the novel space before frailty assessment. There may be other factors that could affect the mouse FI scores. For example, circadian rhythms have been shown to affect a range of behaviours in the mouse model (Chaudhury and Colwell 2002; Loss et al. 2015; Verma et al. 2010) and could affect frailty assessment. Therefore, FI testing should be completed at approximately the same time of day within experiments. The timing in terms of other potential influences should also be considered. Factors such as anaesthesia (Mena et al. 2010) or stress (Mesa-Gresa et al. 2016) or even routine cleaning of animal rooms and cages (Balcombe et al. 2004; Saibaba et al. 1996) are known to affect health and behaviour and may also affect FI scores. Practical considerations, such as making sure the assessment space has 
enough light to properly see small deficits on the mouse (e.g. corneal opacity) are also potentially important.

The housing conditions of the mice to be assessed may also affect aspects of the FI. Garner et al. (2004) found that factors including cage height from the floor, the material the cage was constructed from and whether mice were housed with siblings, significantly affected the amount and severity of barbering. Barbering is a common behaviour seen in laboratory mice, in which mice over-groom the hair or whiskers from themselves or their cage mates (Garner et al. 2004). As hair loss due to barbering or other causes is one of the items in the mouse clinical FI, these housing factors may need to be considered or controlled. Other environmental factors could also influence items in the mouse FI. For example, body composition (e.g. weight, body fat and bone mineral content) differs between mice that are housed singly and in groups (Nagy et al. 2002; Toth 2015). Single versus group housing (Grimm et al. 1996; Morse et al. 1993), whether mice are littermates or not (Hahn and Schanz 1996) and whether mice are virgins or retired breeders (Ingram et al. 1983), affects the results of behavioural tests and could impact items in the FI, although this has not yet been investigated. Similarly, cage temperature, bedding type, noise levels and the presence or absence of environmental enrichment in the cage has been shown to influence experimental outcomes in a variety of different studies (reviewed by Toth 2015). The impact of these environmental and social factors on the development of frailty could be interesting to explore in the mouse model, as these factors are known to affect the development of frailty in humans (Andrew 2015). Such studies in pre-clinical models could shed light on mechanisms underlying development of frailty under different environmental conditions.

\section{Other items that may be included in the mouse clinical FI}


An additional factor that may play a role in the implementation of the mouse clinical FI is the specific items that make up the index. A range of FIs composed of many different sets of items have been used to assess frailty in humans (Rockwood and Mitnitski 2007). Studies have shown that the specific items included in a FI, provided they meet certain criteria, are not important (Rockwood and Mitnitski 2007). The criteria for items to be included in a FI are: 1) that the deficits are health-related; 2) that the deficits generally increase with age; 3) that the FI is composed of more than 30 different items; and 4) that the items in the FI do not saturate (e.g. become apparent in all individuals) at too early an age (Searle et al. 2008). It is possible that the mouse clinical FI could be modified to contain either more or different items if they met these same criteria. For example, Kane and Howlett (2017) suggest that the potential inclusion of cognitive items, such as memory testing (Morris 1984), could be a very useful addition to the battery of evaluations in the mouse clinical FI. Social or behavioural assessments, such as whether the mice are singly housed, or nest making assessments (Deacon 2006; Kalliokoski et al. 2014), also could be added to the FI assessment tool (Kane and Howlett 2017). Some of the items assessed in the Parks et al. (2012) index, including abnormal blood test results (e.g. $\mathrm{Na}^{+}$, $\mathrm{K}^{+}$, urea, glucose etc.), or open field activity measurements (e.g. distance moved, average speed, rearing frequency etc.), could be easily included in the mouse clinical FI. Functional assessments, such as those proposed by Liu et al. (2014) in a mouse frailty phenotype model, also could be included in the mouse FI.

The addition or replacement of items in the mouse FI could be particularly useful in optimising the FI for use in other mouse strains. For example, Kane et al. (2016a) used the clinical FI to assess frailty in the rapidly aging DBA/2J mouse strain (Yuan et al. 2011). However, they found that all the DBA/2J mice were deaf by 2-3 weeks of age, resulting in 
saturation of the hearing loss deficit, so this item could be replaced in an FI specifically tailored for use in this strain. It also would be possible to optimise the specific deficits in frailty assessments to develop FIs for use in a range of different mouse strains including other rapidly aging strains (Gurkar and Niedernhofer 2015) and outbred mouse strains (Chia et al. 2005). It is also possible that the concept of assessing frailty as FI deficit accumulation could be applied to develop novel frailty screening tools for other species used in aging research. Our group recently modified the mouse clinical FI for use in Fischer 344 rats (Yorke et al. 2017). Some of the deficits were removed, for example vision loss, as this item saturates at a young age for all albino rats, and some items were added, such as porphyrin staining which is commonly seen in ageing Fischer 344 rats (Yorke et al. 2017). Approaches to develop such tools for other commonly used models of aging such as nematodes (Caenorhabditis elegans) and fruit flies (Drosophila melanogaster) have been recently proposed by our group (Kane and Howlett 2017).

\section{Conclusions}

The mouse FI is a novel and valuable preclinical model of frailty with applications across a wide range of frailty research areas. It has been used to explore the effect of interventions, both positive and negative, on frailty. It has also been used to explore the effect of frailty on outcomes including drug toxicity, changes to the microbiome and atrial dysfunction. The mouse clinical FI has good overall inter-rater reliability, with the sex and experience of the rater with the assessment tool having no effect on FI score. Care may need to be given to choosing raters of the same professional background, however, if more than one rater is to be compared, and in controlling for other observer factors such as mouse handling technique, and blinding for treatment groups. Environmental factors such as timing and location of assessment, and housing conditions of the mice may also effect FI scores but further studies are needed. Finally, specific 
items in the mouse FI can be replaced or supplemented, especially in the optimisation of the FI for other strains or species as we have recently shown in the aging rat model (Yorke et al. 2017).

\section{REFERENCES}

Andrew, M.K. 2015. Frailty and Social Vulnerability. Interdiscip. Top. Gerontol. Geriatr. 41:186-195. doi: 10.1159/000381236

Balcombe, J., Barnard, N., and Sandusky, C. 2004. Laboratory routines cause animal stress. Contemp. Top. Lab. Anim. Sci. 43:42-51.

Baur, J., Pearson, K.J., Price, N.L., Jamieson, H.A., Lerin, C., Kalra, A., et al. 2006. Resveratrol improves health and survival of mice on a high-calorie diet. Nature, 444:337-42. doi: $10.1038 /$ nature 05354

Burgess, C.L., Holman, C.D.J., and Satti, A.G. 2005. Adverse drug reactions in older Australians 1981-2002. Med. J. Aust. 182:267-270.

Buta, B.J., Walston, J.D., Godino, J.G., Park, M., Kalyani R.R., Xue, Q., et al. 2016. Frailty assessment instruments: Systematic characterization of the uses and contexts of highly-cited instruments. Ageing Res. Rev. 26:53-61. doi: 10.1016/j.arr.2015.12.003

Chaudhury, D., and Colwell, C.S. 2002. Circadian modulation of learning and memory in fearconditioned mice. Behav. Brain Res. 133:95-108. doi: 10.1016/S0166-4328(01)00471-5

Chia, R., Achilli, F., Festing, M.F., and Fisher, E.M. 2005. The origins and uses of mouse outbred stocks. Nat. Genet. 37:1181-1186. doi: ng1665 [pii] \r10.1038/ng1665 
Clegg, A., Young, J., Iliffe, S., Rikkert, M.O., and Rockwood, K. 2013. Frailty in elderly people. Lancet, 381:752-62. doi: 10.1016/S0140-6736(12)62167-9

Collard, R.M., Boter, H., Schoevers, R.A., and Oude Voshaar, R.C. 2012. Prevalence of frailty in community-dwelling older persons: A systematic review. J. Am. Geriatr. Soc. 60:1487-1492. doi: $10.1111 / \mathrm{j} .1532-5415.2012 .04054 . \mathrm{x}$

Deacon, R.M.J. 2006. Assessing nest building in mice. Nat. Protoc. 1:1117-9. doi: $10.1038 /$ nprot. 2006.170

Feridooni, H.A., Sun, M.H., Rockwood, K., and Howlett, S.E. 2015. Reliability of a Frailty Index Based on the Clinical Assessment of Health Deficits in Male C57BL/6J Mice. J. Gerontol. Ser. A Biol. Sci. Med. Sci. 70:686-93. doi: 10.1093/gerona/glu161

Garner, J.P., Dufour, B., Gregg, L.E., Weisker, S.M., and Mench, J.A. 2004. Social and husbandry factors affecting the prevalence and severity of barbering ("whisker trimming") by laboratory mice. Appl. Anim. Behav. Sci. 89:263-282. doi: 10.1016/j.applanim.2004.07.004

Gnjidic, D., Hilmer, S.N., Blyth, F.M., Naganathan, V., Cumming, R.G., Handelsman, D.J., et al. 2012. High-risk prescribing and incidence of frailty among older community-dwelling men. Clin. Pharmacol. Ther. 91:521-8. doi: 10.1038/clpt.2011.258

Grimm, M.S., Emerman, J.T., and Weinberg, J. 1996. Effects of social housing condition and behavior on growth of the Shionogi mouse mammary carcinoma. Physiol. Behav. 59:633-642. doi: 10.1016/0031-9384(95)02126-4

Gurkar, A.U., and Niedernhofer, L.J. 2015. Comparison of mice with accelerated aging caused by distinct mechanisms. Exp. Gerontol. 68:43-50. doi: 10.1016/j.exger.2015.01.045 
Hahn, M.E., and Schanz, N. 1996. Issues in the genetics of social behavior: Revisited. Behav. Genet. 26:463-470. doi: 10.1007/BF02359750

Harris, R., and Dyson, E. 2001. Recruitment of frail older people to research: lessons learnt through experience. J. Adv. Nurs. 36:643-51.

Howlett, S. 2015. Assessment of Frailty in Animal Models. In Frailty in Aging. Biological, Clinical and Social Implications. Edited by O. Theou and K. Rockwood. Karger, Basel, pp 1525

Howlett, S.E., Rockwood, M.R.H., Mitnitski, A., and Rockwood, K. 2014. Standard laboratory tests to identify older adults at increased risk of death. BMC Med. 12:171. doi: 10.1186/s12916014-0171-9

Hubbard, R.E., and Theou, O. 2012. Frailty: enhancing the known knowns. Age Ageing, 41:574575. doi: 10.1093/ageing/afs093

Huizer-Pajkos, A., Kane, A.E., Howlett, S.E., Mach, J., Mitchell, S.J., de Cabo, R., et al. 2016. Adverse Geriatric Outcomes Secondary to Polypharmacy in a Mouse Model: The Influence of Aging. J. Gerontol. Ser. A Biol. Sci. Med. Sci. 71:571-7. doi: 10.1093/gerona/glv046

Ingram, D.K., Spangler, E.L., and Vincent, G.P. 1983. Behavioral comparison of aged virgin and retired breeder mice. Exp. Aging Res. 9:111-113.

Kalliokoski, O., Teilmann, A.C., Jacobsen, K.R., Abelson K.S.P., and Hau, J. 2014. The lonely mouse - Single housing affects serotonergic signaling integrity measured by 8-OH-DPATinduced hypothermia in male mice. PLoS One, 9:1-13. doi: 10.1371/journal.pone.0111065 
Kane, A., Hilmer, S., Boyer, D., Gavin, K., Nines, D., Howlett, S.E. et al. 2016a. Impact of Longevity Interventions on a Validated Mouse Clinical Frailty Index. J. Gerontol. Ser. A Biol. Sci. Med. Sci. 71:333-9. doi: 10.1093/gerona/glu315

Kane, A., Hilmer, S., Mach, J., Mitchell, S.J., de Cabo, R., and Howlett, S.E. 2016b. Animal models of frailty: current applications in clinical research. Clin. Interv. Aging, 2016 (11): 15191529.

Kane, A., and Howlett, S. 2017. Approaches to the assessment of frailty in animal models. In: Handbook of Models for Human Aging, 2nd Edition. In Press.

Kane, A., Mitchell, S.J., Mach, J., Huizer-Pajkos, A., McKenzie, C., Jones, B., et al. $2016 c$. Acetaminophen hepatotoxicity in mice: Effect of age, frailty and exposure type. Exp. Gerontol. 73:95-106. doi: 10.1016/j.exger.2015.11.013

Kane, A.E., Hilmer, S.N., Huizer-Pajkos, A., Mach, J., Nines, D., Boyer, D., et al. 2015. Factors that Impact on Interrater Reliability of the Mouse Clinical Frailty Index. J. Gerontol. Ser. A Biol. Sci. Med. Sci. 70:694-695. doi: 10.1093/gerona/glv032

Langille, M., Meehan, C.J., Koenig, J.E., Dhanani, A.S., Rose, R.A, Howlett, S.E., et al. 2016. Microbial shifts in the aging mouse gut. Microbiome, 2:50. doi: 10.1186/s40168-014-0050-9

Liu, H., Graber, T.G., Ferguson-Stegall, L., and Thompson, L.V. 2014. Clinically Relevant Frailty Index for Mice. J. Gerontol. Ser. A Biol. Sci. Med. Sci. 69:1485-9. doi: $10.1093 /$ gerona/glt188 
Loss, C.M., Binder, L.B., Muccini, E., Martins, W.C., de Oliveira, P.A., Vandresen-Filho, S., et al. 2015. Influence of environmental enrichment vs. time-of-day on behavioral repertoire of male albino Swiss mice. Neurobiol. Learn. Mem. 125:63-72. doi: 10.1016/j.nlm.2015.07.016

Macleod, M.R., McLean, A.L, Kyriakopoulou, A., Serghiou, S., de Wilde, A., Sherratt, N., et al 2015. Risk of Bias in Reports of In Vivo Research: A Focus for Improvement. PLoS Biol. 13:112. doi: 10.1371/journal.pbio.1002273

Mena, M.Á., Perucho, J., Rubio, I., and De Yébenes, J.G. 2010. Studies in animal models of the effects of anesthetics on behavior, biochemistry, and neuronal cell death. J. Alzheimer's Dis. 22 (Suppl. 3):43-48. doi: 10.3233/JAD-2010-100822

Mesa-Gresa, P., Ramos-Campos, M., and Redolat, R. 2016. Corticosterone levels and behavioral changes induced by simultaneous exposure to chronic social stress and enriched environments in NMRI male mice. Physiol. Behav. 158:6-17. doi: 10.1016/j.physbeh.2016.02.027

Mitchell, S.J., Kane, A.E. and Hilmer, S.N. 2011. Age-Related Changes in the Hepatic Pharmacology and Toxicology of Paracetamol. Curr. Gerontol. Geriatr. Res. 2011:14. doi: $10.1155 / 2011 / 624156$

Mitnitski, A.B., Mogilner, A.J., MacKnight, C., and Rockwood, K. 2002. The mortality rate as a function of accumulated deficits in a frailty index. Mech. Ageing Dev. 123:1457-60.

Mitnitski, A.B., Mogilner, A.J., and Rockwood, K. 2001. Accumulation of Deficits as a Proxy Measure of Aging. Sci. World J. 1:323-336. doi: 10.1100/tsw.2001.58 
Moghtadaei, M., Jansen, H.J., Mackasey, M., Rafferty, S.A., Bogachev, O., Sapp, J.L., et al. 2016. The impacts of age and frailty on heart rate and sinoatrial node function. J. Physiol. 594(23): 7105-7126. doi: 10.1113/JP272979

Morris, R. 1984. Developments of a water-maze procedure for studying spatial learning in the rat. J. Neurosci. Methods, 11:47-60.

Morse, A.C., Erwin, V.G., and Jones, B.C. 1993. Strain and housing affect cocaine self-selection and open-field locomotor activity in mice. Pharmacol. Biochem. Behav. 45:905-912.

Nagy, T.R., Krzywanski, D., Li, J., Meleth, S., and Desmond, R. 2002. Effect of group vs. single housing on phenotypic variance in C57BL/6J mice. Obes. Res. 10:412-415. doi: 10.1038/oby.2002.57

Novak, J., Bailoo, J.D., Melotti, L., Rommen, J., and Wurbe, H. 2015. An exploration based cognitive bias test for mice: Effects of handling method and stereotypic behaviour. PLoS One 10(7): e0130718. doi: 10.1371/journal.pone.0130718

Parks, R.J., Fares, E., Macdonald, J.K., Ernst, M.C., Sinal, C.J., Rockwood, K. et al. 2012. A procedure for creating a frailty index based on deficit accumulation in aging mice. J. Gerontol. Ser. A Biol. Sci. Med. Sci. 67:217-227. doi: 10.1093/gerona/glr193

Rockwood, K., Blodgett, J.M., Theou, O., Sun, M.H., Feridooni, H.A., Mitnitsi, A., et al. 2017. A Frailty Index Based On Deficit Accumulation Quantifies Mortality Risk in Humans and in Mice. Sci. Rep. 7: Article Number 43068. doi:10.1038/srep43068

Rockwood, K., Fox, R.A., Stolee, P., Robertson, D. and Beattie, B.L. 1994. Frailty in elderly people: An evolving concept. CMAJ 150:489-495. 
Rockwood, K., and Mitnitski, A. 2007. Frailty in relation to the accumulation of deficits. J. Gerontol. Ser. A Biol. Sci. Med. Sci. 62(7):722-727. doi: 10.1093/gerona/62.7.722

Rockwood, K., Song, X., and Mitnitski, A.B. 2011. Changes in relative fitness and frailty across the adult lifespan: evidence from the Canadian National Population Health Survey. Can. Med. Assoc. J. 138:487-94. doi: 10.1503/cmaj.110626

Saibaba, P., Sales, G., Stodulski, G., and Hau, J. 1996. Behaviour of rats in their home cages: daytime variations and effects of routine husbandry procedures analysed by time sampling techniques. Lab. Anim. 30:13-21.

Searle, S.D., Mitnitski, A., Gahbauer, E.A., Gill, T.M., and Rockwood, K. 2008. A standard procedure for creating a frailty index. BMC Geriatrics 2008, 8:24. doi: 10.1186/1471-2318-8-24

Seldeen, K.L., Pang, M., and Troen, B.R. 2015. Mouse Models of Frailty: an Emerging Field. Curr. Osteoporos. Rep. 13:280-286. doi: 10.1007/s11914-015-0283-y

Sorge, R.E., Martin, L.J., Isbester, K.A., Sotocinal, S.G., Rosen, S., Tuttle, A.H., et al. 2014. Olfactory exposure to males, including men, causes stress and related analgesia in rodents. Nat. Methods, 11:629-632. doi: 10.1038/nmeth.2935

Streiner, D.L., Norman, G.R., and Cairney, J. 2015. Health Measurement Scales: A practical guide to their development and use, Fifth Ed. Oxford Univ Press, Oxford, UK

Toth, L.A. 2015. The influence of the cage environment on rodent physiology and behavior: Implications for reproducibility of pre-clinical rodent research. Exp. Neurol. 270:72-77. doi: 10.1016/j.expneurol.2015.04.010 
Verma, P., Hellemans, K.G.C., Choi, F.Y., Yu, W., and Weinberg, J. 2010. Circadian phase and sex effects on depressive/anxiety-like behaviors and HPA axis responses to acute stress. Physiol. Behav. 99:276-285. doi: 10.1016/j.physbeh.2009.11.002

Weindruch, R., Walford, R.L., Fligiel, S., and Guthrie, D. 1986. The retardation of aging in mice by dietary restriction: longevity, cancer, immunity and lifetime energy intake. J. Nutr. 116:64154.

Whitehead, J.C., Hildebrand, B.A., Sun, M., Rockwood, M.R., Rose, R.A., Rockwood, K., and Howlett, S.E. 2014. A clinical frailty index in aging mice: comparisons with frailty index data in humans. J. Gerontol. Ser. A Biol. Sci. Med. Sci. 69:621-32. doi: 10.1093/gerona/glt136

Yorke, A., Kane, A.E., Hancock Friesen, C.L., Howlett, S.E. and O’Blenes, A. 2017. Development of a Rat Clinical Frailty Index. J. Gerontol. Ser. A Biol. Sci. Med. Sci. doi:10.1093/gerona/glw339

Yuan, R., Peters, L.L., and Paigen, B. 2011. Mice as a mammalian model for research on the genetics of aging. ILAR J. 52:4-15.

Zglinicki, T.V., Nieto, I.V., Brites, D., Karagianni, N., Ortolano, S., Georgopoulos, S. et al. 2016. Frailty in mouse ageing: A conceptual approach. Mech. Ageing Dev. 160: 34-40. doi: 10.1016/j.mad.2016.07.004 
TABLES

Table 1: Specific items and scoring for the 31-item mouse clinical FI

\begin{tabular}{|c|c|c|c|c|}
\hline \multirow[t]{2}{*}{${ }^{1}$ System } & \multirow[t]{2}{*}{ Domain } & \multicolumn{3}{|c|}{ Score } \\
\hline & & $\mathbf{0}$ & 0.5 & 1.0 \\
\hline \multirow[t]{5}{*}{ Integument } & Alopecia & & & \\
\hline & Loss of fur colour & & & \\
\hline & Dermatitis & & & \\
\hline & Loss of whiskers & & & \\
\hline & Coat condition & & & \\
\hline \multirow[t]{8}{*}{ Physical/Musculoskeletal } & Tumours & & & \\
\hline & Distended abdomen & & & \\
\hline & Kyphosis & & & \\
\hline & Tail stiffening & & & \\
\hline & Gait disorders & & & \\
\hline & Tremor & & & \\
\hline & Forelimb grip strength & & & \\
\hline & Body condition score & & & \\
\hline \multirow[t]{2}{*}{ Vestibulocochlear/Auditory } & Vestibular disturbance & & & \\
\hline & Hearing loss & & & \\
\hline \multirow[t]{8}{*}{ Ocular/Nasal } & Cataracts & & & \\
\hline & Corneal opacity & & & \\
\hline & Eye discharge/swelling & & & \\
\hline & Microphthalmia & & & \\
\hline & Vision loss & & & \\
\hline & Menace reflex & & & \\
\hline & Nasal discharge & & & \\
\hline & Malocclusions & & & \\
\hline \multirow[t]{3}{*}{ Digestive/Urogenital } & Rectal prolapse & & & \\
\hline & Vaginal/uterine/penile prolapse & & & \\
\hline & Diarrhoea & & & \\
\hline Respiratory & Breathing rate/depth & & & \\
\hline \multirow[t]{3}{*}{ Discomfort } & Mouse Grimace Scale & & & \\
\hline & Piloerection & & & \\
\hline & $\mathbf{0}$ & 0.25 & 0.5 & 0.75 \\
\hline \multirow[t]{2}{*}{ Other } & Temperature & & & \\
\hline & Weight & & & \\
\hline
\end{tabular}

${ }^{1}$ Frailty assessment form, modified from the original published by Whitehead et al. (2014). The first 29 items are scored as follows: 0 for no deficit, 0.5 for a mild deficit and 1 for a severe deficit. The last two items (temperature and weight) are scored between 0 and 1 (in increments of 0.25 ) based on how many standard deviations they are from the mean reference values obtained in healthy young adult mice. 
Table 2: Inter-rater reliability of the mouse clinical FI

\begin{tabular}{ccc}
\hline & Correlation Coefficient (r) & ICC $^{\mathbf{1}}$ \\
\hline All 4 Raters & - & $0.80(0.68-0.88)$ \\
\hline Experienced vs Inexperienced & 0.70 & $0.76(0.57-0.87)$ \\
\hline Male vs Female & & $0.86(0.74-0.92)$ \\
${ }^{1}$ ICC $=$ Intra-class correlation coefficient. & 0.75 &
\end{tabular}




\section{FIGURE LEGENDS}

Figure 1: FI scores increased with age but were not affected by sex of the observer (A) and positive correlations were observed between male and female observers (B). C57BL/6 mice were assessed using the mouse clinical FI by 4 raters (one male experienced, one female experience, one male inexperienced, one female inexperienced) over a 2 week period. (A) Mean FI scores were calculated for the different mouse age groups (young, $38.7 \pm 2.3$ weeks, $n=15$; middle aged, $60.7 \pm 5.4$ weeks, $n=24$; old, $101.4 \pm 19.4$ weeks, $n=6$ ) for the different sexes of the observers. A 2-way ANOVA showed a significant effect of age $(p<0.001)$, but not of observer sex $(p=0.84)$ on mean FI score. (B) Mean FI scores were calculated for all mice $(n=45)$ for the different observer sexes and correlated against one another to assess agreement between observers. The line in each graph represents a linear regression.

Figure 2. FI scores increased with age but were not affected by experience of the observer (A) and positive correlations were observed between experienced and inexperienced observers (B). C57BL/6 mice were assessed using the mouse clinical FI by 4 raters (one male experienced, one female experience, one male inexperienced, one female inexperienced) over a 2 week period. (A) Mean FI scores were calculated for the different mouse age groups (young, $38.7 \pm 2.3$ weeks, $n=15$; middle aged, $60.7 \pm 5.4$ weeks, $n=24$; old, $101.4 \pm 19.4$ weeks, $n=6$ ) for the different experience levels of the observers. A 2-way ANOVA showed a significant effect of age $(p<0.001)$, but not of observer experience $(p=0.47)$ on mean FI score. (B) Mean FI scores were calculated for all mice $(n=45)$ for the different experience levels and correlated against one another to assess agreement between observers. The line in each graph represents a linear regression. 

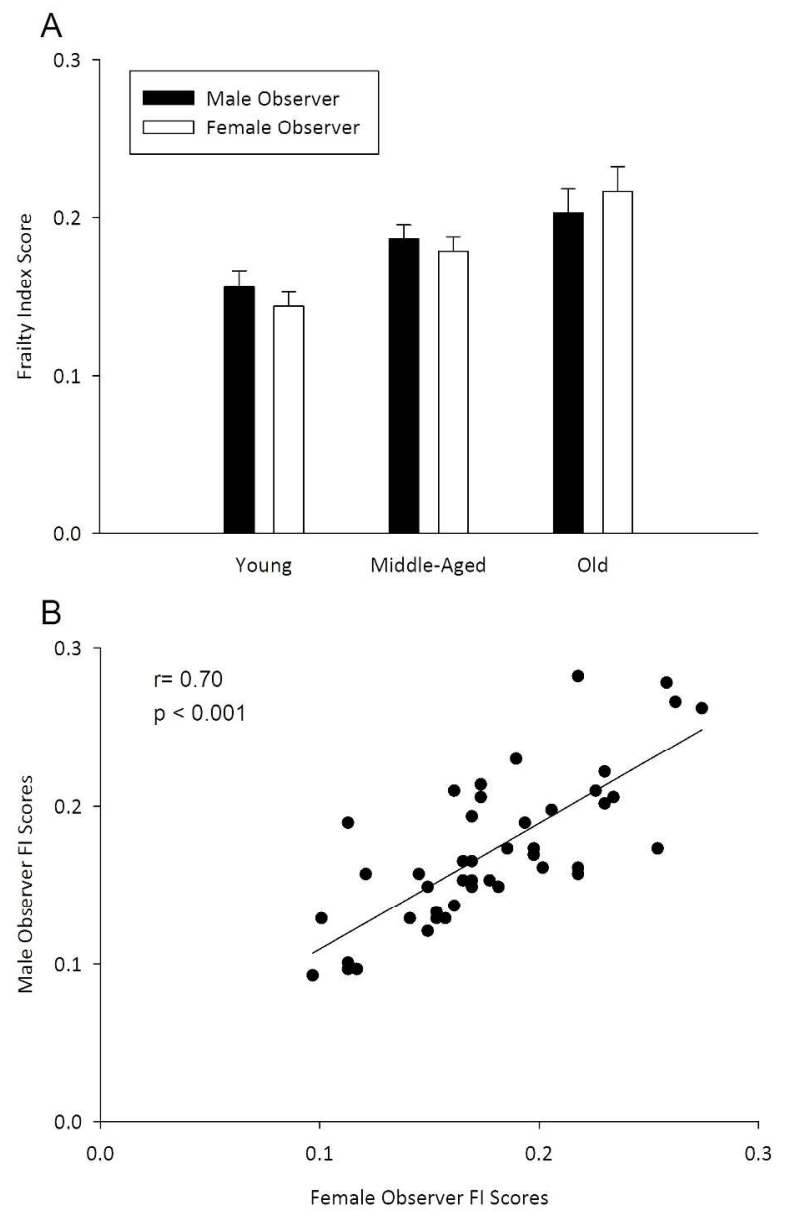

Figure 1: FI scores increased with age but were not affected by sex of the observer $(A)$ and positive correlations were observed between male and female observers (B). C57BL/ 6 mice were assessed using the mouse clinical FI by 4 raters (one male experienced, one female experience, one male inexperienced, one female inexperienced) over a 2 week period. (A) Mean FI scores were calculated for the different mouse age groups (young, $38.7 \pm 2.3$ weeks, $n=15$; middle aged, $60.7 \pm 5.4$ weeks, $n=24$; old, $101.4 \pm 19.4$ weeks, $n=6)$ for the different sexes of the observers. A 2-way ANOVA showed a significant effect of age $(p<0.001)$, but not of observer sex $(p=0.84)$ on mean FI score. (B) Mean FI scores were calculated for all mice $(n=45)$

for the different observer sexes and correlated against one another to assess agreement between observers. The line in each graph represents a linear regression.

$$
210 \times 296 \mathrm{~mm}(300 \times 300 \mathrm{DPI})
$$



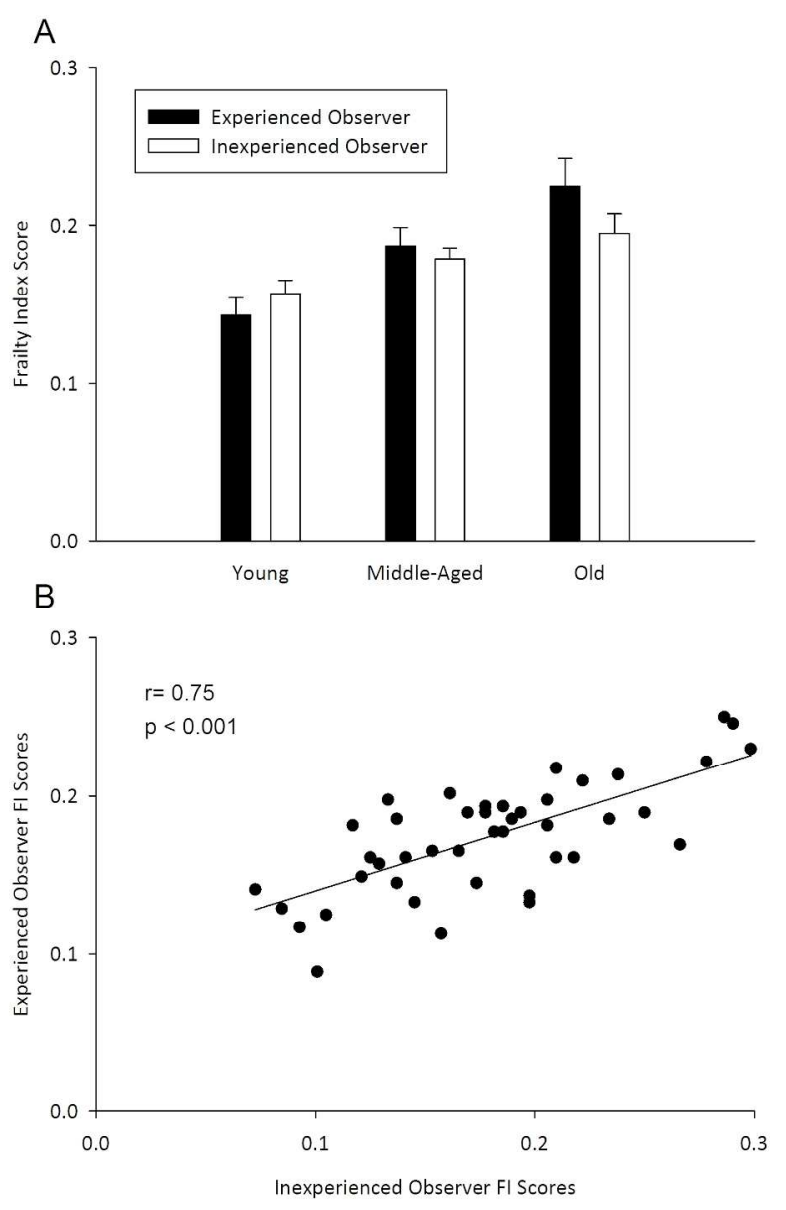

Figure 2. FI scores increased with age but were not affected by experience of the observer $(A)$ and positive correlations were observed between experienced and inexperienced observers (B). C57BL/6 mice were assessed using the mouse clinical FI by 4 raters (one male experienced, one female experience, one male inexperienced, one female inexperienced) over a 2 week period. (A) Mean FI scores were calculated for the different mouse age groups (young, $38.7 \pm 2.3$ weeks, $n=15$; middle aged, $60.7 \pm 5.4$ weeks, $n=24$; old, $101.4 \pm 19.4$ weeks, $n=6$ ) for the different experience levels of the observers. A 2-way ANOVA showed a significant effect of age $(p<0.001)$, but not of observer experience $(p=0.47)$ on mean FI score. (B) Mean FI scores were calculated for all mice $(n=45)$ for the different experience levels and correlated against one another to assess agreement between observers. The line in each graph represents a linear regression.

$$
210 \times 296 \mathrm{~mm}(300 \times 300 \mathrm{DPI})
$$

